Acta Crystallographica Section E

Structure Reports

Online

ISSN 1600-5368

\section{Nicotinium nitrate monohydrate}

\section{Samuel Robinson Jebas, ${ }^{\mathrm{a}}$ Thailampillai Balasubramanian $^{\mathbf{b}}$ * and Mark E. Light ${ }^{c}$}

aDepartment of Electronics, St Joseph's College, Tiruchirappalli 620 002, India, bepartment of Physics, National Institute of Technology, Tiruchirappalli 620 015, India, and ${ }^{\mathbf{c}}$ School of Chemistry, University of Southampton,

Highfield SO17 1BJ, England

Correspondence e-mail: bala@nitt.edu

\section{Key indicators}

Single-crystal X-ray study

$T=120 \mathrm{~K}$

Mean $\sigma(\mathrm{C}-\mathrm{C})=0.004 \AA$

$R$ factor $=0.054$

$w R$ factor $=0.137$

Data-to-parameter ratio $=11.9$

For details of how these key indicators were automatically derived from the article, see http://journals.iucr.org/e.
In the title compound, $\mathrm{C}_{6} \mathrm{H}_{6} \mathrm{NO}_{2}{ }^{+} \cdot \mathrm{NO}_{3}{ }^{-} \cdot \mathrm{H}_{2} \mathrm{O}$, the nicotinium cation is essentially planar. $\mathrm{N}-\mathrm{H} \cdots \mathrm{O}, \mathrm{O}-\mathrm{H} \cdots \mathrm{O}$ and $\mathrm{C}-$ $\mathrm{H}$. . O hydrogen bonds link the molecules into layers parallel to the $(10 \overline{1})$ plane.

\section{Comment}

Nicotinic acid (vitamin B3), known as niacin, is a lipid lowering agent widely used to treat hypertriglyceridemia by the inhibition of lipolysis in adipose tissue (Athimoolam \& Rajaram, 2005). The nicotinic acid complex 5-methylpyrazine2-carboxylic acid-4-oxide is a commonly used drug for the treatment of hypercholesterolemia (Lorenzen et al., 2001). Coordination complexes of nicotinic acid with metals such as Sn possess antitumour activity greater than the well known cisplatin or doxorubicin (Gielen et al., 1992). The enzyme nicotinic acid mononucleotide adenyltransferase is essential for the synthesis of nicotinamide adenine dinucleotide in all living cells and is a potential target for antibiotics (Kim et al., 2004). As a part of our investigation of inorganic salts of nicotinic acid, we report here the crystal structure of nicotinium nitrate monohydrate, (I).

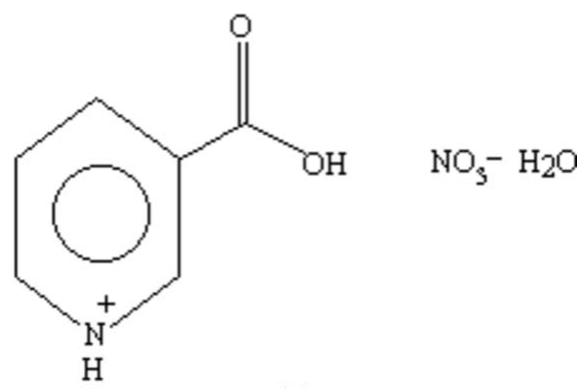

(I)

The asymetric unit of (I) contains a nicotinium cation, a nitrate anion and a water molecule (Fig. 1). Protonation of atom $\mathrm{N} 1$ of nicotine results in a widening of the $\mathrm{C} 2-\mathrm{N} 1-\mathrm{C} 6$ angle to $122.9(3)^{\circ}$, compared with $118.9(3)^{\circ}$ in unprotonated nicotinic acid (Kutoglu \& Scheringer, 1983). The nicotinium cation is essentially planar, with a maximum deviation from the mean plane of 0.048 (2) $\AA$ for atom O1.

The crystal packing is stabilized by $\mathrm{N}-\mathrm{H} \cdots \mathrm{O}, \mathrm{O}-\mathrm{H} \cdots \mathrm{O}$ and $\mathrm{C}-\mathrm{H} \cdots \mathrm{O}$ hydrogen bonds (Table 1), which link the

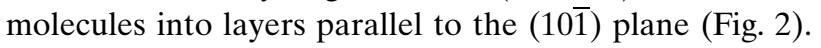

\section{Experimental}

Nitric acid was added dropwise to an aqueous solution of nicotinic acid, in stoichiometric amounts. The solution was heated at $323 \mathrm{~K}$ for
Received 4 July 2006 Accepted 15 July 2006 
$2 \mathrm{~h}$. Colourless block-shaped crystals of (I) were obtained by slow evaporation over a period of one week.

\section{Crystal data \\ $\mathrm{C}_{6} \mathrm{H}_{6} \mathrm{NO}_{2}{ }^{+} \cdot \mathrm{NO}_{3}{ }^{-} \cdot \mathrm{H}_{2} \mathrm{O}$ \\ $M_{r}=204.14$ \\ Monoclinic, $P 2_{1} / n$ \\ $a=6.6539$ (7) $\AA$ \\ $b=12.3682(15) \AA$ \\ $c=10.1814(15) \AA$ \\ $\beta=100.967(7)^{\circ}$ \\ $V=822.59(18) \AA^{3}$}

$$
\begin{aligned}
& Z=4 \\
& D_{x}=1.641 \mathrm{Mg} \mathrm{m}^{-3} \\
& \text { Mo } K \alpha \text { radiation } \\
& \mu=0.15 \mathrm{~mm}^{-1} \\
& T=120(2) \mathrm{K} \\
& \text { Block, colourless } \\
& 0.2 \times 0.2 \times 0.07 \mathrm{~mm}
\end{aligned}
$$

\section{Data collection}

Bruker Nonius KappaCCD areadetector diffractometer

$\varphi$ and $\omega$ scans

Absorption correction: multi-scan (SADABS; Sheldrick, 2003)

$T_{\min }=0.970, T_{\max }=0.990$

\section{Refinement}

Refinement on $F^{2}$

$R\left[F^{2}>2 \sigma\left(F^{2}\right)\right]=0.055$

$w R\left(F^{2}\right)=0.137$

$S=0.98$

1604 reflections

135 parameters
6153 measured reflections 1604 independent reflections 894 reflections with $I>2 \sigma(I)$ $R_{\text {int }}=0.109$

$\theta_{\max }=26.1^{\circ}$

$\mathrm{H}$ atoms treated by a mixture of independent and constrained refinement

$w=1 /\left[\sigma^{2}\left(F_{\mathrm{o}}^{2}\right)+(0.0639 P)^{2}\right]$

where $P=\left(F_{\mathrm{o}}{ }^{2}+2 F_{\mathrm{c}}{ }^{2}\right) / 3$

$(\Delta / \sigma)_{\max }<0.001$

$\Delta \rho_{\max }=0.26 \mathrm{e}^{-3}$

$\Delta \rho_{\min }=-0.30$ e $\AA^{-3}$

\section{Table 1}

Hydrogen-bond geometry $\left(\AA \stackrel{\circ}{\circ}^{\circ}\right.$.

\begin{tabular}{lllll}
\hline$D-\mathrm{H} \cdots A$ & $D-\mathrm{H}$ & $\mathrm{H} \cdots A$ & $D \cdots A$ & $D-\mathrm{H} \cdots A$ \\
\hline $\mathrm{N} 1-\mathrm{H} 1 \cdots \mathrm{O} 3^{\mathrm{i}}$ & 0.86 & 1.93 & $2.782(3)$ & 170 \\
$\mathrm{O} 1-\mathrm{H} 1 A \cdots \mathrm{O} 6$ & 0.82 & 1.77 & $2.587(3)$ & 180 \\
$\mathrm{O} 6-\mathrm{H} 6 A \cdots \mathrm{O} 5$ & $0.93(5)$ & $1.92(5)$ & $2.843(3)$ & $171(4)$ \\
$\mathrm{O} 6-\mathrm{H} 6 B \cdots \mathrm{O} 3^{\mathrm{ii}}$ & $0.88(5)$ & $1.96(5)$ & $2.825(3)$ & $173(5)$ \\
$\mathrm{C} 2-\mathrm{H} 2 \cdots 2^{\text {iii }}$ & 0.93 & 2.43 & $3.173(4)$ & 137 \\
$\mathrm{C} 4-\mathrm{H} 4 \cdots 1^{\text {iv }}$ & 0.93 & 2.46 & $3.262(4)$ & 144 \\
$\mathrm{C} 6-\mathrm{H} 6 \cdots 5^{\text {i }}$ & 0.93 & 2.35 & $3.051(4)$ & 132 \\
$\mathrm{C} 6-\mathrm{H} 6 \cdots 4^{\text {iv }}$ & 0.93 & 2.32 & $3.013(4)$ & 131 \\
\hline
\end{tabular}

Symmetry codes: (i) $x, y-1, z$; (ii) $x-\frac{1}{2},-y+\frac{3}{2}, z-\frac{1}{2}$; (iii) $x+\frac{1}{2},-y+\frac{1}{2}, z+\frac{1}{2}$; (iv) $x-\frac{1}{2},-y+\frac{1}{2}, z-\frac{1}{2}$.

Water $\mathrm{H}$ atoms were located in a difference map and refined freely $[\mathrm{O}-\mathrm{H}=0.88(5)$ and $0.93(5) \AA]$. All other $\mathrm{H}$ atoms were placed in calculated positions, with $\mathrm{C}-\mathrm{H}=0.93 \AA, \mathrm{O}-\mathrm{H}=0.82 \AA$ and $\mathrm{N}-\mathrm{H}=$ $0.86 \AA$, and refined using a riding model, with $U_{\text {iso }}(\mathrm{H})=1.2 U_{\text {eq }}(\mathrm{C}, \mathrm{N})$ and $1.5 U_{\text {eq }}(\mathrm{O})$.

Data collection: COLLECT (Nonius, 1998); cell refinement: $D E N Z O$ (Otwinowski \& Minor, 1997) and COLLECT; data reduction: DENZO and COLLECT; program(s) used to solve structure: SHELXS97 (Sheldrick, 1997); program(s) used to refine structure: SHELXL97 (Sheldrick, 1997); molecular graphics: PLATON (Spek, 2003); software used to prepare material for publication: SHELXL97.

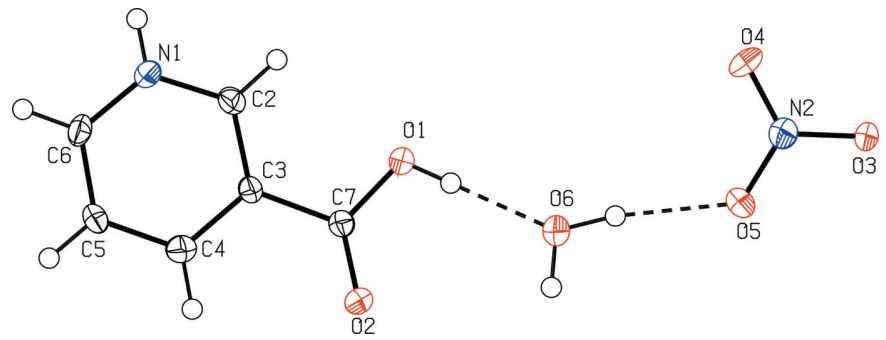

Figure 1

The asymmetric unit of (I), showing the atom-numbering scheme, with $50 \%$ probability displacement ellipsoids. Hydrogen bonds are drawn as dashed lines.

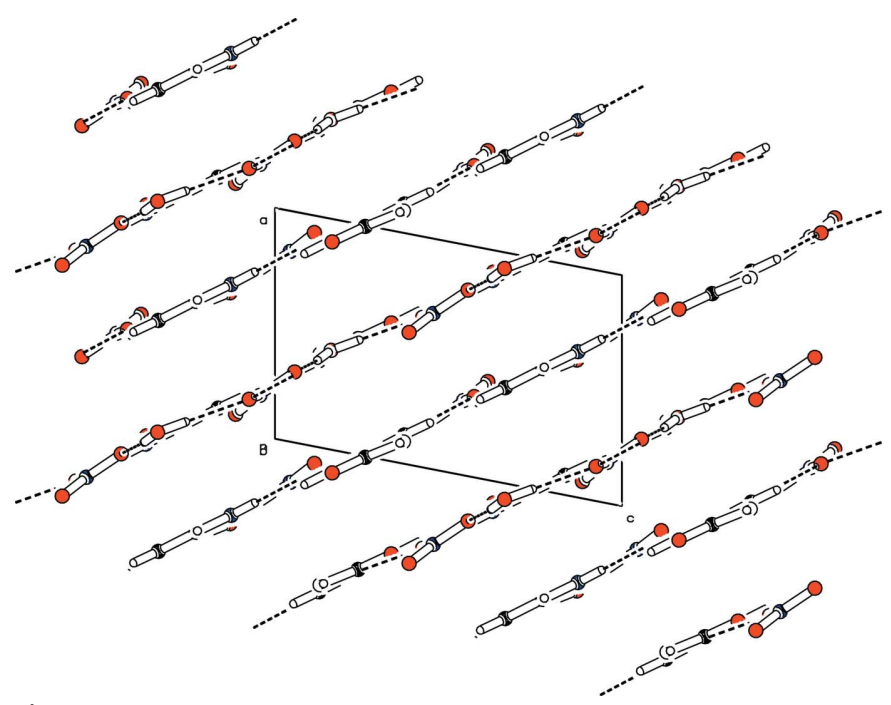

Figure 2

A packing diagram for (I), viewed down the $b$ axis. Hydrogen bonds are drawn as dashed lines.

\section{References}

Athimoolam, S. \& Rajaram, R. K. (2005). Acta Cryst. E61, o2764-o2767.

Gielen, M., Khloufi, A. E., Biesemans, M. \& Willem, R. (1992). Polyhedron, 11, 1861-1868.

Kim, H.-L., Yoon, H.-J., Ha, J. Y., Lee, B. I., Lee, H. H., Mikami, B. \& Suh, S. W. (2004). Acta Cryst. D60, 948-949.

Kutoglu, A. \& Scheringer, C. (1983). Acta Cryst. C39, 232-234.

Lorenzen, A., Stannek, C., Lang, H., Andrianov, V., Kalvinsh, I. \& Schwabe, U. (2001). Mol. Pharmacol. 59, 349-357.

Nonius (1998). COLLECT. Nonius BV, Delft, The Netherlands.

Otwinowski, Z. \& Minor, W. (1997). Methods in Enzymology, Vol. 276, Macromolecular Crystallography, Part A, edited by C. W. Carter Jr \& R. M. Sweet, pp. 307-326. New York: Academic Press.

Sheldrick, G. M. (1997). SHELXL97 and SHELXS97. University of Gottingen, Germany.

Sheldrick, G. M. (2003). SADABS. Version 2.10. University of Gottingen, Germany.

Spek, A. L. (2003). J. Appl. Cryst. 36, 7-13. 


\section{supporting information}

Acta Cryst. (2006). E62, o3481-o3482 [https://doi.org/10.1107/S1600536806027474]

Nicotinium nitrate monohydrate

Samuel Robinson Jebas, Thailampillai Balasubramanian and Mark E. Light

Nicotinium nitrate monohydrate

Crystal data

$\mathrm{C}_{6} \mathrm{H}_{6} \mathrm{NO}_{2}^{+} \cdot \mathrm{NO}_{3}^{-} \cdot \mathrm{H}_{2} \mathrm{O}$

$F(000)=424$

$M_{r}=204.14$

Monoclinic, $P 2_{1} / n$

Hall symbol: -P $2 \mathrm{yn}$

$a=6.6539(7) \AA$

$b=12.3682(15) \AA$

$c=10.1814(15) \AA$

$D_{\mathrm{x}}=1.641 \mathrm{Mg} \mathrm{m}^{-3}$

$\beta=100.967(7)^{\circ}$

$V=822.59(18) \AA^{3}$

Mo $K \alpha$ radiation, $\lambda=0.71073 \AA$

Cell parameters from 25 reflections

$\theta=1.0-26.0^{\circ}$

$\mu=0.15 \mathrm{~mm}^{-1}$

$T=120 \mathrm{~K}$

Block, colourless

$Z=4$

$0.2 \times 0.2 \times 0.07 \mathrm{~mm}$

\section{Data collection}

Bruker-Nonius FR591 rotating anode diffractometer

$\varphi$ and $\omega$ scans

Absorption correction: multi-scan

(SADABS; Sheldrick, 2003)

$T_{\min }=0.970, T_{\max }=0.990$

6153 measured reflections

1604 independent reflections

894 reflections with $I>2 \sigma(I)$

$R_{\text {int }}=0.109$

$\theta_{\max }=26.1^{\circ}, \theta_{\min }=3.3^{\circ}$

$h=-8 \rightarrow 8$

$k=-14 \rightarrow 14$

$l=-12 \rightarrow 9$

3 standard reflections every 60 reflections

intensity decay: none

Refinement

Refinement on $F^{2}$

Least-squares matrix: full

$R\left[F^{2}>2 \sigma\left(F^{2}\right)\right]=0.055$

$w R\left(F^{2}\right)=0.137$

$S=0.98$

1604 reflections

135 parameters

0 restraints

$\mathrm{H}$ atoms treated by a mixture of independent and constrained refinement

$w=1 /\left[\sigma^{2}\left(F_{\mathrm{o}}^{2}\right)+(0.0639 P)^{2}\right]$

where $P=\left(F_{\mathrm{o}}^{2}+2 F_{\mathrm{c}}{ }^{2}\right) / 3$

$(\Delta / \sigma)_{\max }<0.001$

$\Delta \rho_{\max }=0.26 \mathrm{e} \AA^{-3}$

$\Delta \rho_{\min }=-0.30$ e $\AA^{-3}$

\section{Special details}

Geometry. All e.s.d.'s (except the e.s.d. in the dihedral angle between two 1.s. planes) are estimated using the full covariance matrix. The cell e.s.d.'s are taken into account individually in the estimation of e.s.d.'s in distances, angles and torsion angles; correlations between e.s.d.'s in cell parameters are only used when they are defined by crystal symmetry. An approximate (isotropic) treatment of cell e.s.d.'s is used for estimating e.s.d.'s involving 1.s. planes. 
Fractional atomic coordinates and isotropic or equivalent isotropic displacement parameters $\left(\AA^{2}\right)$

\begin{tabular}{|c|c|c|c|c|}
\hline & $x$ & $y$ & $z$ & $U_{\text {iso }} * / U_{\text {eq }}$ \\
\hline O1 & $0.0945(3)$ & $0.36210(16)$ & $0.3701(2)$ & $0.0259(6)$ \\
\hline H1A & 0.0866 & 0.4279 & 0.3602 & $0.039 *$ \\
\hline $\mathrm{O} 2$ & $-0.0994(3)$ & $0.36163(16)$ & $0.1648(2)$ & $0.0258(6)$ \\
\hline N1 & $0.1318(3)$ & 0.03157 (19) & $0.3733(3)$ & $0.0205(7)$ \\
\hline H1 & 0.2044 & -0.001 & 0.4406 & $0.025 *$ \\
\hline $\mathrm{C} 2$ & 0.1259 (4) & $0.1401(2)$ & $0.3736(3)$ & $0.0196(7)$ \\
\hline $\mathrm{H} 2$ & 0.1984 & 0.1786 & 0.4458 & $0.023 *$ \\
\hline $\mathrm{C} 3$ & $0.0112(4)$ & $0.1940(2)$ & 0.2659 & $0.0167(7)$ \\
\hline $\mathrm{C} 4$ & -0.0953 & $0.1334(2)$ & $0.1597(3)$ & $0.0211(8)$ \\
\hline $\mathrm{H} 4$ & -0.1732 & 0.168 & 0.0861 & $0.025^{*}$ \\
\hline $\mathrm{C} 5$ & $-0.0851(4)$ & $0.0216(2)$ & 0.1637 (3) & $0.0219(8)$ \\
\hline H5 & -0.1556 & -0.0191 & 0.0929 & $0.026^{*}$ \\
\hline C6 & 0.0292 (4) & $-0.0283(2)$ & $0.2724(3)$ & $0.0209(8)$ \\
\hline H6 & 0.0361 & -0.1034 & 0.2764 & $0.025 *$ \\
\hline $\mathrm{C} 7$ & $-0.0042(4)$ & $0.3138(2)$ & $0.2603(3)$ & $0.0181(7)$ \\
\hline N2 & $0.3245(4)$ & $0.8028(2)$ & $0.5438(3)$ & $0.0235(7)$ \\
\hline $\mathrm{O} 3$ & 0.3502 & $0.90208(16)$ & 0.5744 & $0.0243(6)$ \\
\hline O4 & $0.4266(3)$ & $0.73309(17)$ & $0.6135(2)$ & $0.0342(7)$ \\
\hline O5 & 0.1940 & 0.77703 (17) & $0.4435(2)$ & $0.0268(6)$ \\
\hline O6 & 0.0695 & $0.56951(18)$ & $0.3383(3)$ & $0.0264(6)$ \\
\hline H6A & $0.124(6)$ & $0.634(4)$ & $0.377(4)$ & $0.067(14)^{*}$ \\
\hline H6B & $0.002(6)$ & $0.584(4)$ & $0.258(5)$ & $0.082(17)^{*}$ \\
\hline
\end{tabular}

Atomic displacement parameters $\left(\AA^{2}\right)$

\begin{tabular}{lllllll}
\hline & $U^{11}$ & $U^{22}$ & $U^{33}$ & $U^{12}$ & $U^{13}$ & $U^{23}$ \\
\hline O1 & $0.0344(12)$ & $0.0150(12)$ & $0.0236(14)$ & $0.0002(10)$ & $-0.0062(10)$ & $-0.0002(10)$ \\
O2 & $0.0352(12)$ & $0.0176(12)$ & $0.0210(13)$ & $0.0032(10)$ & $-0.0040(10)$ & $0.0015(10)$ \\
N1 & $0.0219(14)$ & $0.0155(14)$ & $0.0227(16)$ & $0.0005(11)$ & $0.0003(11)$ & $0.0030(12)$ \\
C2 & $0.0169(16)$ & $0.0196(17)$ & $0.0207(18)$ & $-0.0013(13)$ & $-0.0002(13)$ & $-0.0022(14)$ \\
C3 & $0.0182(16)$ & $0.0153(17)$ & $0.0163(18)$ & $-0.0003(12)$ & $0.0023(13)$ & $-0.0026(13)$ \\
C4 & $0.0198(16)$ & $0.0225(18)$ & $0.0204(19)$ & $0.0011(13)$ & $0.0021(13)$ & $0.0017(15)$ \\
C5 & $0.0252(17)$ & $0.0186(18)$ & $0.0200(18)$ & $-0.0050(14)$ & $-0.0008(13)$ & $-0.0035(14)$ \\
C6 & $0.0237(17)$ & $0.0125(16)$ & $0.0265(19)$ & $-0.0012(13)$ & $0.0048(14)$ & $0.0011(14)$ \\
C7 & $0.0187(16)$ & $0.0168(16)$ & $0.0179(18)$ & $-0.0021(13)$ & $0.0015(14)$ & $0.0003(14)$ \\
N2 & $0.0266(15)$ & $0.0199(16)$ & $0.0235(17)$ & $-0.0015(12)$ & $0.0037(13)$ & $0.0010(13)$ \\
O3 & $0.0339(12)$ & $0.0131(12)$ & $0.0227(13)$ & $-0.0011(10)$ & $-0.0027(9)$ & $0.0000(10)$ \\
O4 & $0.0420(14)$ & $0.0201(13)$ & $0.0351(16)$ & $0.0088(11)$ & $-0.0065(12)$ & $0.0080(11)$ \\
O5 & $0.0318(13)$ & $0.0226(12)$ & $0.0225(14)$ & $-0.0040(10)$ & $-0.0039(11)$ & $-0.0027(10)$ \\
O6 & $0.0303(13)$ & $0.0179(13)$ & $0.0276(15)$ & $-0.0025(10)$ & $-0.0027(11)$ & $0.0000(11)$ \\
& & & & & & \\
\hline
\end{tabular}

Geometric parameters $\left(A,{ }^{\circ}\right)$

\begin{tabular}{llll}
\hline $\mathrm{O} 1-\mathrm{C} 7$ & $1.326(3)$ & $\mathrm{C} 4-\mathrm{C} 5$ & $1.384(4)$ \\
$\mathrm{O} 1-\mathrm{H} 1 \mathrm{~A}$ & 0.82 & $\mathrm{C} 4-\mathrm{H} 4$ & 0.93
\end{tabular}




\begin{tabular}{|c|c|c|c|}
\hline $\mathrm{O} 2-\mathrm{C} 7$ & $1.209(3)$ & $\mathrm{C} 5-\mathrm{C} 6$ & $1.365(4)$ \\
\hline $\mathrm{N} 1-\mathrm{C} 2$ & $1.342(4)$ & $\mathrm{C} 5-\mathrm{H} 5$ & 0.93 \\
\hline $\mathrm{N} 1-\mathrm{C} 6$ & $1.343(4)$ & $\mathrm{C} 6-\mathrm{H} 6$ & 0.93 \\
\hline $\mathrm{N} 1-\mathrm{H} 1$ & 0.86 & $\mathrm{~N} 2-\mathrm{O} 4$ & $1.235(3)$ \\
\hline $\mathrm{C} 2-\mathrm{C} 3$ & $1.383(4)$ & $\mathrm{N} 2-\mathrm{O} 5$ & $1.249(3)$ \\
\hline $\mathrm{C} 2-\mathrm{H} 2$ & 0.93 & $\mathrm{~N} 2-\mathrm{O} 3$ & $1.271(3)$ \\
\hline $\mathrm{C} 3-\mathrm{C} 4$ & $1.393(4)$ & O6- $\mathrm{H} 6 \mathrm{~A}$ & $0.93(5)$ \\
\hline $\mathrm{C} 3-\mathrm{C} 7$ & $1.486(4)$ & O6- $-\mathrm{H} 6 \mathrm{~B}$ & $0.88(5)$ \\
\hline $\mathrm{C} 7-\mathrm{O} 1-\mathrm{H} 1 \mathrm{~A}$ & 109.5 & $\mathrm{C} 6-\mathrm{C} 5-\mathrm{C} 4$ & $119.5(3)$ \\
\hline $\mathrm{C} 2-\mathrm{N} 1-\mathrm{C} 6$ & $122.9(3)$ & $\mathrm{C} 6-\mathrm{C} 5-\mathrm{H} 5$ & 120.2 \\
\hline $\mathrm{C} 2-\mathrm{N} 1-\mathrm{H} 1$ & 118.6 & $\mathrm{C} 4-\mathrm{C} 5-\mathrm{H} 5$ & 120.2 \\
\hline $\mathrm{C} 6-\mathrm{N} 1-\mathrm{H} 1$ & 118.6 & $\mathrm{~N} 1-\mathrm{C} 6-\mathrm{C} 5$ & $119.6(3)$ \\
\hline $\mathrm{N} 1-\mathrm{C} 2-\mathrm{C} 3$ & $119.4(3)$ & $\mathrm{N} 1-\mathrm{C} 6-\mathrm{H} 6$ & 120.2 \\
\hline $\mathrm{N} 1-\mathrm{C} 2-\mathrm{H} 2$ & 120.3 & $\mathrm{C} 5-\mathrm{C} 6-\mathrm{H} 6$ & 120.2 \\
\hline $\mathrm{C} 3-\mathrm{C} 2-\mathrm{H} 2$ & 120.3 & $\mathrm{O} 2-\mathrm{C} 7-\mathrm{O} 1$ & $123.9(3)$ \\
\hline $\mathrm{C} 2-\mathrm{C} 3-\mathrm{C} 4$ & $118.6(3)$ & $\mathrm{O} 2-\mathrm{C} 7-\mathrm{C} 3$ & $122.7(3)$ \\
\hline $\mathrm{C} 2-\mathrm{C} 3-\mathrm{C} 7$ & $122.3(3)$ & $\mathrm{O} 1-\mathrm{C} 7-\mathrm{C} 3$ & $113.4(2)$ \\
\hline $\mathrm{C} 4-\mathrm{C} 3-\mathrm{C} 7$ & $119.0(3)$ & $\mathrm{O} 4-\mathrm{N} 2-\mathrm{O} 5$ & $120.8(2)$ \\
\hline $\mathrm{C} 5-\mathrm{C} 4-\mathrm{C} 3$ & $119.9(3)$ & $\mathrm{O} 4-\mathrm{N} 2-\mathrm{O} 3$ & $120.0(2)$ \\
\hline $\mathrm{C} 5-\mathrm{C} 4-\mathrm{H} 4$ & 120 & $\mathrm{O} 5-\mathrm{N} 2-\mathrm{O} 3$ & $119.2(2)$ \\
\hline $\mathrm{C} 3-\mathrm{C} 4-\mathrm{H} 4$ & 120 & $\mathrm{H} 6 \mathrm{~A}-\mathrm{O} 6-\mathrm{H} 6 \mathrm{~B}$ & $108(4)$ \\
\hline $\mathrm{C} 6-\mathrm{N} 1-\mathrm{C} 2-\mathrm{C} 3$ & $-0.5(5)$ & $\mathrm{C} 2-\mathrm{N} 1-\mathrm{C} 6-\mathrm{C} 5$ & $0.8(5)$ \\
\hline $\mathrm{N} 1-\mathrm{C} 2-\mathrm{C} 3-\mathrm{C} 4$ & $0.0(5)$ & $\mathrm{C} 4-\mathrm{C} 5-\mathrm{C} 6-\mathrm{N} 1$ & $-0.6(5)$ \\
\hline $\mathrm{N} 1-\mathrm{C} 2-\mathrm{C} 3-\mathrm{C} 7$ & $179.3(3)$ & $\mathrm{C} 2-\mathrm{C} 3-\mathrm{C} 7-\mathrm{O} 2$ & $177.8(3)$ \\
\hline $\mathrm{C} 2-\mathrm{C} 3-\mathrm{C} 4-\mathrm{C} 5$ & $0.1(5)$ & $\mathrm{C} 4-\mathrm{C} 3-\mathrm{C} 7-\mathrm{O} 2$ & $-2.9(5)$ \\
\hline $\mathrm{C} 7-\mathrm{C} 3-\mathrm{C} 4-\mathrm{C} 5$ & $-179.2(3)$ & $\mathrm{C} 2-\mathrm{C} 3-\mathrm{C} 7-\mathrm{O} 1$ & $-2.8(4)$ \\
\hline $\mathrm{C} 3-\mathrm{C} 4-\mathrm{C} 5-\mathrm{C} 6$ & $0.2(5)$ & $\mathrm{C} 4-\mathrm{C} 3-\mathrm{C} 7-\mathrm{O} 1$ & $176.5(3)$ \\
\hline
\end{tabular}

Hydrogen-bond geometry $\left(\AA,{ }^{\circ}\right)$

\begin{tabular}{lllll}
\hline$D-\mathrm{H} \cdots A$ & $D-\mathrm{H}$ & $\mathrm{H} \cdots A$ & $D \cdots A$ & $D-\mathrm{H} \cdots A$ \\
\hline $\mathrm{N} 1-\mathrm{H} 1 \cdots \mathrm{O} 3^{\mathrm{i}}$ & 0.86 & 1.93 & $2.782(3)$ & 170 \\
$\mathrm{O} 1-\mathrm{H} 1 A \cdots \mathrm{O} 6$ & 0.82 & 1.77 & $2.587(3)$ & 180 \\
$\mathrm{O} 6-\mathrm{H} 6 A \cdots \mathrm{O} 5$ & $0.93(5)$ & $1.92(5)$ & $2.843(3)$ & $171(4)$ \\
$\mathrm{O} 6-\mathrm{H} 6 B \cdots \mathrm{O} 3^{\text {ii }}$ & $0.88(5)$ & $1.96(5)$ & $2.825(3)$ & $173(5)$ \\
$\mathrm{C} 2-\mathrm{H} 2 \cdots \mathrm{O} 2^{\mathrm{iii}}$ & 0.93 & 2.43 & $3.173(4)$ & 137 \\
$\mathrm{C} 4-\mathrm{H} 4 \cdots 1^{\text {iv }}$ & 0.93 & 2.46 & $3.262(4)$ & 144 \\
$\mathrm{C} 6-\mathrm{H} 6 \cdots 5^{\mathrm{i}}$ & 0.93 & 2.35 & $3.051(4)$ & 132 \\
$\mathrm{C} 6-\mathrm{H} 6 \cdots{ }^{\mathrm{iv}}$ & 0.93 & 2.32 & $3.013(4)$ & 131
\end{tabular}

Symmetry codes: (i) $x, y-1, z$; (ii) $x-1 / 2,-y+3 / 2, z-1 / 2$; (iii) $x+1 / 2,-y+1 / 2, z+1 / 2$; (iv) $x-1 / 2,-y+1 / 2, z-1 / 2$. 